\title{
Computer Configuration
}

National Cancer Institute

\section{Source}

National Cancer Institute. Computer Configuration. NCI Thesaurus. Code C85235.

Refers to 1) the way a computer's operating system is set up, 2) the total combination of hardware components that make up the computer system, or 3) the specific software settings that have been established for the system. 